\title{
Checklist dos peixes de água doce do Estado de São Paulo, Brasil
}

\author{
Osvaldo Takeshi Oyakawa ${ }^{1,2}$ \& Naércio Aquino Menezes ${ }^{1}$ \\ ${ }^{1}$ Museu de Zoologia, Universidade de São Paulo - USP, \\ Av. Nazaré, n. 481, CEP 04263-000, São Paulo, SP, Brasil \\ ${ }^{2}$ Autor para correspondência: Osvaldo Takeshi Oyakawa, e-mail: oyakawa@usp.br
}

OYAKAWA, O.T. \& MENEZES, N.A. Checklist of fresh water fishes from São Paulo State, Brazil. Biota Neotrop. 11(1a): http://www.biotaneotropica.org.br/v11n1a/en/abstract?inventory+bn0021101a2011.

\begin{abstract}
The fish species living in the freshwaters of the São Paulo state (393) are distributed among four major river basins: upper Paraná, Paraíba do Sul, Ribeira de Iguape and a set of small coastal drainages flowing directly into the Atlantic Ocean. Since these river basins drain areas with distinct vegetation types, soils, etc., each one has a different species composition. In the Upper Paraná the large Rio Paraná and some of its large tributaries (Tietê, Paranapanema, and Grande) contain large species that support commercial fisheries, however, 70 to $80 \%$ of the ichthyofauna is composed by small species found in small streams, including those in headwaters where many are endemic. The inventory of the ichthyofauna greatly benefited from three research projects supported by the BIOTA/FAPESP program, but much work remains to be done to collect and describe new species from areas such as deep channels, headwaters and marginal and swampy áreas around ponds and man-made lakes and reservoirs. The ichthyofauna of the São Paulo State has suffered from deforestation, sewage, damming of rivers for construction of power plants, urbanization, etc., so that 66 species are currently considered threatened at various levels according to the criteria of the International Union for Conservation of Nature and Natural Resources (IUCN).

Keywords: fresh water fishes, biodiversity of the State of São Paulo, BIOTA/FAPESP Program.
\end{abstract}

Number of species: In the world: 28,000, in Brazil: 2,587, estimated in São Paulo State: 393.

OYAKAWA, O.T. \& MENEZES, N.A. Checklist dos peixes de água doce do Estado de São Paulo, Brasil. Biota Neotrop. 11(1a): http://www.biotaneotropica.org.br/v11n1a/pt/abstract?inventory+bn0021101a2011.

Resumo: As espécies de peixes de água doce encontradas no estado de São Paulo distribuem-se entre quatro bacias hidrográficas principais: Alto Paraná, Paraíba do Sul, Ribeira de Iguape e um conjunto de pequenas drenagens costeiras que desembocam diretamente no oceano Atlântico. Como estas bacias drenam áreas com diferentes tipos de vegetação, solos, etc., cada uma tem uma composição de espécies diferente. No Alto Paraná, o grande Rio Paraná e alguns de seus maiores afluentes (Tietê, Paranapanema e Grande) possuem espécies de grande porte que sustentam a pesca comercial e de subsistência, mas 70 a $80 \%$ da ictiofauna é composta por espécies de pequeno porte de pequenos riachos, incluindo os de cabeceira, onde muitas são endêmicas. O inventário da ictiofauna foi incrementado através de três projetos de pesquisa apoiados pelo programa BIOTA/FAPESP, mas ainda resta muito trabalho de coleta e descrição de novas espécies de áreas pouco exploradas, como calhas de rios, regiões de cabeceiras, alagadiços em áreas marginais de reservatórios e lagos. A ictiofauna do Estado de São Paulo tem sofrido da ação deletéria de poluição, desmatamento, esgotos urbanos e construção de reservatórios para produção de energia elétrica, etc., de tal forma que atualmente 66 espécies são consideradas ameaçadas em vários níveis, de acordo com os critérios da "International Union for Conservation of Nature and Natural Resources" (IUCN). Palavras-chave: peixes de água doce, biota paulista, Programa BIOTA/FAPESP.

Número de espécies: No mundo: 28.000, no Brasil: 2.587, estimadas no Estado de São Paulo: 393. 


\section{Introdução}

As águas doces do Estado de São Paulo concentram-se em quatro bacias hidrográficas: Alto Paraná, Paraíba do Sul, Ribeira de Iguape e um conjunto de pequenas drenagens situadas numa estreita faixa litorânea que fluem diretamente para o oceano Atlântico e compõem as Drenagens Costeiras ou Bacia Litorânea. O Alto Paraná, a maior das quatro bacias hidrográficas, inclui também parte dos estados de Minas Gerais, Goiás, Mato Grosso do Sul, Paraná além do Distrito Federal; o Ribeira de Iguape drena também parte das terras do Paraná; e o Paraíba do Sul partes de Minas Gerais e Rio de Janeiro. A bacia do Alto Paraná está quase que inteiramente incluída no bioma do Cerrado, com exceção da sub-bacia do Alto Tietê que, desde a sua nascente em Salesópolis até Pirapora do Bom Jesus e Santana do Parnaíba, drena áreas de Mata Atlântica. As outras três bacias hidrográficas que drenam o estado estão no bioma da Mata Atlântica (Oyakawa et al. 2009).

Estima-se que existam cerca de 55.000 espécies de vertebrados em todo o mundo, das quais aproximadamente 28.000 são peixes (Nelson 2006). Nas águas doces neotropicais existem atualmente 4.475 espécies válidas e cerca de 1.550 espécies ainda não descritas segundo estimativa recente, totalizando 6.025 espécies nestes ambientes (Reis et al. 2003); deste total o Brasil abriga 2.587 espécies, segundo Buckup et al. (2007).

\section{Metodologia}

A lista apresentada na Tabela 1 é resultado da consulta a diferentes fontes (Reis et al. 2003, Buckup et al. 2007, Langeani et al. 2007, Oyakawa et al. 2009, Eschmeyer 2010), artigos recentes de revisões e descrições de novas espécies de peixes do Estado de São Paulo e exame da coleção de peixes do Museu de Zoologia da Universidade de São Paulo. Na lista foram consideradas, indistintamente, para cada bacia hidrográfica, as espécies autóctones ou nativas (que ocorrem naturalmente em cada uma das quatro bacias), as alóctones (espécies originárias de outras bacias hidrográficas da Região Neotropical e introduzidas nas bacias hidrográficas do Estado de São Paulo) e exóticas (espécies originárias de outros continentes).

Calcular com precisão o número de espécies de peixes de água doce restritas ao Estado de São Paulo é uma tarefa difícil uma vez que, como mencionado na Introdução, as quatro bacias hidrográficas do estado compartilham seus limites com outros estados vizinhos à São Paulo o que dificulta sobremaneira a delimitação de limites geopolíticos das espécies de peixes. Nesse sentido, a bacia do Alto Paraná é a mais complexa, pois seus limites abrangem cinco estados diferentes.

\section{Resultados e Discussão}

No estado de São Paulo existem cerca de 391 espécies, o que corresponde a aproximadamente $15 \%$ do total estimado para todo o território brasileiro (Tabela 1). Desse total, 260 espécies ocorrem no Alto Paraná, 97 no Ribeira de Iguape, 71 no Paraíba do Sul e 57 na Bacia Litorânea, algumas espécies podendo ocorrer em duas ou mais bacias diferentes. As espécies estão distribuídas em 10 ordens e 39 famílias, sendo Characidae com 83 e Loricariidae com 81 as mais numerosas.

O Rio Paraná e seus principais afluentes (Tietê, Paranapanema e Grande) possuem cursos d'água de maior porte que ainda abrigam várias espécies de médio e grande porte como os curimbatás, piaparas, pintados e jaús que apresentam ampla distribuição geográfica e importância econômica na pesca comercial e de subsistência. As bacias do Ribeira de Iguape, Paraíba do Sul e as drenagens costeiras não possuem espécies de grande porte e migradoras como o Alto
Paraná, com exceção, talvez, da presença de Hoplias lacerdae e Hypostomus tapijara, espécies de grande porte, na bacia do Ribeira de Iguape; a primeira representada por exemplares capturados recentemente com $85 \mathrm{~cm}$ e a segunda por exemplares até $50 \mathrm{~cm}$ de comprimento total.

Além desses cursos maiores existem, em todas as quatro bacias hidrográficas do estado, um incontável número de riachos e ambientes de cabeceiras habitados por espécies pequenas, com até 10 a $15 \mathrm{~cm}$ de comprimento, de distribuição restrita, com pouco ou nenhum valor comercial (exceto de algumas poucas espécies exploradas no comércio de peixes ornamentais) e com grande dependência da vegetação ripária como fonte de itens alimentares de origem alóctone, para reprodução e proteção (Castro \& Menezes 1998). É possível afirmar que entre 70 a $80 \%$ das espécies do Estado de São Paulo são de pequeno porte e habitam preferencialmente esses ambientes de riacho. Dentre as 36 espécies de peixes descritas do Estado de São Paulo no período de 2000 a 2010, somente duas, Hypostomus tapijara e Sternarchorhynchus britskii, ocorrem em habitats fora de ambientes de riachos e cabeceiras. Hypostomus tapijara Oyakawa, Akama \& Zanata, 2005, é conhecida somente dos rios maiores da bacia do Ribeira de Iguape e Sternarchorhynchus britskii Campos da Paz, 2000, do reservatório de Ilha Solteira, no Rio Paraná.

Um aspecto importante a ser ressaltado foi o apoio do Programa BIOTA/FAPESP à inventariação da fauna de peixes de água doce das bacias do Alto Paraná e do Ribeira de Iguape nos últimos doze anos, que possibilitou a descoberta da grande maioria das espécies descritas nesse período. Três projetos foram subsidiados pelo Programa: "Diversidade de peixes de riachos e cabeceiras da bacia do Alto Paraná no Estado de São Paulo coordenado por Ricardo Macedo Corrêa e Castro; "Diversidade de Peixes de Riachos e Cabeceiras da Bacia do Rio Ribeira de Iguape no estado de São Paulo, coordenado por Osvaldo Takeshi Oyakawa e "A Ictiofauna do Alto Paraná", coordenado por Francisco Langeani Neto. O projeto "Conhecimento," conservação e utilização racional da diversidade da fauna de peixes do Brasil" - PRONEX/CNPq, coordenado por Naércio Aquino Menezes também contribuiu para inventariação da fauna do estado, em especial da Bacia do Rio Ribeira de Iguape.

No estado de São Paulo existem atualmente 66 espécies sob ameaça de extinção em diferentes graus (Tabela 1), além de 16 espécies classificadas como DD, ou seja, deficiente em dados, segundo os critérios propostos pela IUCN (International... 2001). Neste caso, a grande maioria das espécies também são de pequeno porte, típicas de riachos de cabeceiras, sujeitas à poluição dos corpos d'água por esgotos industriais e domésticos, assoreamento e destruição da mata ripária.

Grandes peixes migradores do Alto Paraná, como os pacus, jaús, pintados e surubins sofrem também com a fragmentação dos habitats resultante da construção de inúmeras barragens hidrelétricas de grande porte nos maiores rios da bacia. Além destas, outras menores conhecidas como Pequenas Centrais Hidrelétricas (PCH) também têm sido construídas nos rios de menor porte, causando os mesmos efeitos nocivos sobre a ictiofauna. Um desses empreendimentos atualmente sendo construído no Rio Paraíba do Sul, quase na divisa com o Estado do Rio de Janeiro, vai comprometer seriamente um dos únicos trechos de corredeira e grandes poços de pedra do rio no Estado de São Paulo, habitat do surubim-do-paraíba (Steindachneridion parahybae).

Espécies de peixes restritas à bacia litorânea, formada por pequenos rios que drenam diretamente para o mar, também sofrem os impactos negativos da urbanização desenfreada que provoca a destruição de grandes áreas da mata de restinga. A parte baixa da bacia do Ribeira de Iguape, já na planície litorânea, nos municípios de Iguape e Cananéia, onde existem extensas áreas de restinga, também sofrem com a destruição das matas, assoreamento e poluição de suas águas. 
Tabela 1. Ictiofauna do Estado de São Paulo. (*) Espécies ameaçadas de extinção segundo Oyakawa et al. 2009.

Table 1. Ichthyofauna of São Paulo State. (*) endangered species according to Oyakawa et al. 2009.

\begin{tabular}{|c|c|c|c|c|}
\hline & $\begin{array}{c}\text { Alto } \\
\text { Paraná }\end{array}$ & $\begin{array}{c}\text { Ribeira } \\
\text { de Iguape }\end{array}$ & $\begin{array}{c}\text { Paraíba } \\
\text { do Sul }\end{array}$ & $\begin{array}{c}\text { Bacia } \\
\text { Litorânea }\end{array}$ \\
\hline \multicolumn{5}{|l|}{ Myliobatiformes } \\
\hline \multicolumn{5}{|l|}{ Potamotrygonidae } \\
\hline Potamotrygon falkneri Castex \& Maciel, 1963 & $\mathrm{X}$ & - & - & - \\
\hline Potamotrygon motoro (Müller \& Henle, 1841) & $\mathrm{X}$ & - & - & - \\
\hline \multicolumn{5}{|l|}{ Clupeiformes } \\
\hline \multicolumn{5}{|l|}{ Clupeidae } \\
\hline Platanichthys platana (Regan, 1917) & $\mathrm{X}$ & - & - & - \\
\hline \multicolumn{5}{|l|}{ Cypriniformes } \\
\hline \multicolumn{5}{|l|}{ Cyprinidae } \\
\hline Aristichthys nobilis (Richardson, 1845) & $\mathrm{X}$ & - & - & - \\
\hline Ctenopharyngodon idella (Valenciennes, 1844) & $\mathrm{X}$ & - & - & - \\
\hline Cyprinus carpio Linnaeus, 1758 & $\mathrm{X}$ & - & - & - \\
\hline \multicolumn{5}{|l|}{ Characiformes } \\
\hline \multicolumn{5}{|l|}{ Acestrorhynchidae } \\
\hline Acestrorhynchus lacustris (Lütken, 1875) & $\mathrm{X}$ & - & - & - \\
\hline \multicolumn{5}{|l|}{ Anostomidae } \\
\hline Leporellus vittatus (Valenciennes, 1850) & $\mathrm{X}$ & - & - & - \\
\hline Leporinus aguapeiensis Amaral-Campos, 1945 & $\mathrm{X}$ & - & - & - \\
\hline Leporinus amblyrhynchus Garavello \& Britski, 1987 & $X$ & - & - & - \\
\hline Leporinus conirostris Steindachner, 1875 & - & - & $\mathrm{X}$ & - \\
\hline Leporinus copelandii Steindachner, 1875 & - & - & $\mathrm{X}$ & - \\
\hline Leporinus friderici (Bloch, 1794) & $\mathrm{X}$ & - & - & - \\
\hline Leporinus lacustris Campos, 1945 & $\mathrm{X}$ & - & - & - \\
\hline Leporinus macrocephalus Garavello \& Britski, 1988 & $\mathrm{X}$ & - & - & - \\
\hline Leporinus mormyrops Steindachner, 1875 & - & - & $\mathrm{X}$ & - \\
\hline Leporinus obtusidens (Valenciennes, 1836) & $\mathrm{X}$ & - & - & - \\
\hline Leporinus octofasciatus Steindachner, 1915 & $\mathrm{X}$ & - & - & - \\
\hline Leporinus paranensis Garavello \& Britski, 1987 & $\mathrm{X}$ & - & - & - \\
\hline Leporinus cf. steindachneri Eigenmann, 1097 & - & $\mathrm{X}$ & - & - \\
\hline Leporinus striatus Kner, 1859 & $\mathrm{X}$ & - & - & - \\
\hline Leporinus thayeri Borodin, $1929 *$ & - & - & $\mathrm{X}$ & - \\
\hline Schizodon altoparanae Garavello \& Britski, 1990 & $\mathrm{X}$ & - & - & - \\
\hline Schizodon intermedius Garavello \& Britski, 1990 & $\mathrm{X}$ & - & - & - \\
\hline Schizodon nasutus Kner, 1858 & $\mathrm{X}$ & - & - & - \\
\hline \multicolumn{5}{|l|}{ Characidae } \\
\hline Aphyocharax anisitsi Eigenmann \& Eigenmann, 1903 & $\mathrm{X}$ & - & - & - \\
\hline Aphyocharax dentatus Eigenmann \& Kennedy, 1903 & $\mathrm{X}$ & - & - & - \\
\hline Aphyocheirodon hemigrammus Eigenmann, 1915 & $\mathrm{X}$ & - & - & - \\
\hline Astyanax altiparanae Garutti \& Britski, 2000 & $\mathrm{X}$ & - & - & - \\
\hline Astyanax cf. bimaculatus (Linnaeus, 1758) & - & $\mathrm{X}$ & $\mathrm{X}$ & - \\
\hline Astyanax biotae Castro \& Vari, 2004 & $\mathrm{X}$ & - & - & - \\
\hline Astyanax bockmanni Vari \& Castro, 2007 & $X$ & - & - & - \\
\hline Astyanax fasciatus (Cuvier, 1819) & $\mathrm{X}$ & - & $\mathrm{X}$ & - \\
\hline Astyanax giton Eigenmann, 1908 & - & - & $\mathrm{X}$ & - \\
\hline Astyanax intermedius Eigemann, 1908 & - & - & $\mathrm{X}$ & - \\
\hline Astyanax janeiroensis Eigenmann, 1908 & - & $\mathrm{X}$ & - & $\mathrm{X}$ \\
\hline Astyanax parahybae Eigenmann, 1908 & - & - & $\mathrm{X}$ & - \\
\hline Astyanax paranae Eigenmann, 1914 & $\mathrm{X}$ & - & - & $\mathrm{X}$ \\
\hline
\end{tabular}


Tabela 1. Continuação...

\begin{tabular}{cccc} 
Alto & Ribeira & Paraíba & Bacia \\
Paraná & de Iguape & do Sul & Litorânea \\
\hline
\end{tabular}

Astyanax ribeirae Eigenmann, 1911

Astyanax cf. scabripinnis Eigenmann, 1908

Astyanax schubarti Britski, 1964

Astyanax taeniatus (Jenyns, 1842)

Astyanax trierythropterus Godoy, 1970 *

Brycon amazonicus (Agassiz, 1829)

Brycon hilarii (Valenciennes, 1903)

Brycon insignis Steindachner, 1876 *

Brycon nattereri Günther, 1864 *

Brycon opalinus (Cuvier, 1817) *

Brycon orbignyanus (Valenciennes in Cuvier \& Valenciennes, 1850) *

Bryconamericus exodon Eigenmann, 1907

Bryconamericus iheringii (Boulenger, 1887)

Bryconamericus microcephalus (Ribeiro, 1908)

Bryconamericus stramineus Eigenmann, 1908

Bryconamericus turiuba Langeani; Lucena; Pedrini \& Tarelho-Pereira, 2005

Colossoma macropomum (Cuvier, 1818)

Coptobrycon bilineatus (Ellis, 1911)*

Cynopotomus kincaidi (Schultz, 1950)

Deuterodon iguape Eigenmann, 1907

Deuterodon pedri Eigenmann, 1908

Galeocharax knerii (Steindachner, 1879)

Glandulocauda melanopleura (Ellis, 1911) *

Gymnocorymbus ternetzi (Boulenger, 1895)

Hasemania hanseni (Fowler, 1949)

Hemigrammus marginatus Ellis, 1911

Hemigrammus parana Marinho, Carvalho, Langeani \& Tatsumi, 2008

Hollandichthys multifasciatus (Eigenmann \& Norris, 1900)

Hyphessobrycon anisitsi (Eigenmann, 1907)

Hyphessobrycon balbus Myers, 1927

Hyphessobrycon bifasciatus Ellis, 1911

Hyphessobrycon duragenys Ellis, 1911 *

Hyphessobrycon eques (Steindachner, 1882)

Hyphessobrycon flammeus Myers, 1924 *

Hyphessobrycon griemi Hoedeman, 1957

Hyphessobrycon reticulatus Ellis, 1911

Knodus moenkhausii (Eigenmann \& Kennedy, 1903)

Metynnis maculatus (Kner, 1858)

Metynnis mola Eigenmann \& Kennedy, 1903

Mimagoniates lateralis (Nichols, 1913) *

Mimagoniates microlepis (Steindachner, 1876)

Moenkhausia intermedia Eigenmann, 1908

Moenkhausia sanctaefilomenae (Steindachner, 1907)

Myleus tiete (Eigenmann \& Norris, 1900)*

Mylossoma duriventre (Cuvier, 1818)

Odontostilbe microcephala Eigenmann, 1907

Oligobrycon microstomus Eigenmann, 1915

Oligosarcus hepsetus (Cuvier, 1817)

Oligosarcus paranensis Menezes \& Géry, 1983

\begin{tabular}{|c|c|c|c|}
\hline- & $X$ & - & - \\
\hline$X$ & - & $X$ & $X$ \\
\hline$X$ & - & - & - \\
\hline - & - & $X$ & $X$ \\
\hline$X$ & - & - & - \\
\hline$X$ & - & - & - \\
\hline$X$ & - & - & - \\
\hline- & - & $X$ & - \\
\hline$X$ & - & - & - \\
\hline- & - & $X$ & - \\
\hline$X$ & - & - & - \\
\hline$X$ & - & - & - \\
\hline$X$ & - & - & - \\
\hline- & $X$ & - & - \\
\hline $\mathrm{X}$ & - & - & - \\
\hline $\mathrm{X}$ & - & - & - \\
\hline$X$ & - & - & - \\
\hline$X$ & - & - & $\mathrm{X}$ \\
\hline$X$ & - & - & - \\
\hline - & $X$ & - & $X$ \\
\hline - & - & $X$ & - \\
\hline$X$ & - & - & - \\
\hline$X$ & $X$ & - & $X$ \\
\hline $\mathrm{X}$ & - & - & - \\
\hline $\mathrm{X}$ & - & - & - \\
\hline $\mathrm{X}$ & - & - & - \\
\hline $\mathrm{X}$ & - & - & - \\
\hline$X$ & $X$ & - & $X$ \\
\hline$X$ & - & - & - \\
\hline$X$ & - & - & - \\
\hline$X$ & $X$ & $X$ & - \\
\hline$X$ & $X$ & - & $\mathrm{X}$ \\
\hline$X$ & $X$ & $X$ & - \\
\hline $\mathrm{X}$ & - & - & - \\
\hline - & $X$ & - & $X$ \\
\hline $\mathrm{X}$ & $X$ & $X$ & $X$ \\
\hline$X$ & - & - & - \\
\hline $\mathrm{X}$ & - & - & - \\
\hline $\mathrm{X}$ & - & - & - \\
\hline - & - & - & $X$ \\
\hline$X$ & $X$ & - & - \\
\hline $\mathrm{X}$ & - & - & - \\
\hline $\mathrm{X}$ & - & - & - \\
\hline$X$ & - & - & - \\
\hline$X$ & - & - & - \\
\hline$X$ & - & - & - \\
\hline- & - & $X$ & - \\
\hline - & $X$ & $X$ & - \\
\hline $\mathrm{X}$ & - & - & - \\
\hline
\end{tabular}


Tabela 1. Continuação...

\begin{tabular}{|c|c|c|c|c|}
\hline & $\begin{array}{c}\text { Alto } \\
\text { Paraná }\end{array}$ & $\begin{array}{c}\text { Ribeira } \\
\text { de Iguape }\end{array}$ & $\begin{array}{c}\text { Paraíba } \\
\text { do Sul }\end{array}$ & $\begin{array}{c}\text { Bacia } \\
\text { Litorânea }\end{array}$ \\
\hline Oligosarcus pintoi Campos, 1945 & $\mathrm{X}$ & - & - & - \\
\hline Oligosarcus planaltinae Menezes \& Géy, 1983 & $\mathrm{X}$ & - & - & - \\
\hline Piabina anhembi Silva \& Kaefer, 2003 & $\mathrm{X}$ & - & - & - \\
\hline Piabina argentea Reinhardt, 1867 & $\mathrm{X}$ & - & - & - \\
\hline Piaractus mesopotamicus (Holmberg, 1887) * & $\mathrm{X}$ & - & - & - \\
\hline Planaltina britskii Menezes; Weitzman \& Burns, 2003 & $\mathrm{X}$ & - & - & - \\
\hline Planaltina glandipedis Menezes; Weitzman \& Burns, 2003 & $\mathrm{X}$ & - & - & - \\
\hline Probolodus heterostomus Eigenmann, 1911 & - & $\mathrm{X}$ & $\mathrm{x}$ & - \\
\hline Pseudocorynopoma heterandria Eigenmann, 1914 * & $\mathrm{X}$ & $\mathrm{X}$ & - & - \\
\hline Rachoviscus crassiceps Myers, 1926 * & - & - & - & $\mathrm{X}$ \\
\hline Roeboides descalvadensis Fowler, 1932 & $\mathrm{X}$ & - & - & - \\
\hline Salminus brasiliensis (Cuvier, 1816) & $\mathrm{X}$ & - & $\mathrm{X}$ & - \\
\hline Salminus hilarii Valenciennes, 1850 & $\mathrm{X}$ & - & $\mathrm{X}$ & - \\
\hline Serrapinnus heterodon (Eigenmann, 1915) & $\mathrm{X}$ & - & - & - \\
\hline Serrapinnus notomelas (Eigenmann, 1915) & $\mathrm{X}$ & - & - & - \\
\hline Serrasalmus maculatus Kner, 1858 & $\mathrm{X}$ & - & - & - \\
\hline Serrasalmus marginatus Valenciennes, 1837 & $\mathrm{x}$ & - & - & - \\
\hline Spintherobolus broccae Myers, 1925 * & - & - & - & $\mathrm{X}$ \\
\hline Spintherobolus leptoura Weitzman \& Malabarba, $1999 *$ & - & $\mathrm{X}$ & - & - \\
\hline Spintherobolus papilliferus Eigenmann, $1911 *$ & $\mathrm{X}$ & - & - & - \\
\hline Triportheus nematurus (Kner, 1858) & $\mathrm{x}$ & - & - & - \\
\hline \multicolumn{5}{|l|}{ Crenuchidae } \\
\hline Characidium alipioi Travassos, 1955 & - & - & $\mathrm{X}$ & - \\
\hline Characidium fasciatum Reinhardt, 1866 & $\mathrm{X}$ & - & - & - \\
\hline Characidium gomesi Travassos, 1956 & $\mathrm{X}$ & - & - & - \\
\hline Characidium japuhybense Travassos, 1949 & $\mathrm{X}$ & $\mathrm{X}$ & - & $\mathrm{X}$ \\
\hline Characidium cf. lagosantense Travassos, 1956 & $\mathrm{X}$ & - & - & - \\
\hline Characidium lanei Travassos, 1967 & - & $\mathrm{X}$ & - & $\mathrm{x}$ \\
\hline Characidium laterale (Boulenger, 1895) & $\mathrm{x}$ & - & - & - \\
\hline Characidium lauroi (Travassos, 1949) & $\mathrm{X}$ & - & $\mathrm{X}$ & - \\
\hline Characidium oiticicai Travassos, 1967 & $\mathrm{X}$ & $\mathrm{X}$ & - & $\mathrm{X}$ \\
\hline Characidium pterostictum Gomes, 1947 & - & $\mathrm{x}$ & - & - \\
\hline Characidium schubarti Travassos, 1955 & $\mathrm{X}$ & $\mathrm{X}$ & - & - \\
\hline Characidium zebra Eigenmann, 1909 & $\mathrm{x}$ & - & - & - \\
\hline \multicolumn{5}{|l|}{ Curimatidae } \\
\hline Cyphocharax gilbert (Quoy \& Gaimard, 1824) & - & - & $\mathrm{X}$ & $\mathrm{x}$ \\
\hline Cyphocharax modestus (Fernández-Yépez, 1948) & $\mathrm{x}$ & - & - & - \\
\hline Cyphocharax nagelii (Steindachner, 1881) & $\mathrm{x}$ & - & - & - \\
\hline Cyphocharax santacatarinae (Fernandez-Yepez, 1948) & - & $\mathrm{X}$ & - & $\mathrm{X}$ \\
\hline Cyphocharax vanderi (Britski, 1980) & $\mathrm{x}$ & - & - & - \\
\hline Steindachnerina insculpta (Fernández-Yépez, 1948) & $\mathrm{x}$ & - & - & - \\
\hline \multicolumn{5}{|l|}{ Cynodontidae } \\
\hline Rhaphiodon vulpinus Spix \& Agassiz, 1829 & $\mathrm{x}$ & - & - & - \\
\hline \multicolumn{5}{|l|}{ Erythrinidae } \\
\hline Erythrinus erythrinus (Bloch \& Schneider, 1801) & $\mathrm{x}$ & - & - & - \\
\hline Hoplerythrinus unitaeniatus (Agassiz, 1829) & $\mathrm{X}$ & - & - & - \\
\hline Hoplias intermedius (Günther, 1864) & $\mathrm{x}$ & - & - & - \\
\hline Hoplias lacerdae Miranda-Ribeiro, 1908 * & - & $\mathrm{X}$ & - & - \\
\hline Hoplias cf. malabaricus (Bloch, 1794) & $\mathrm{x}$ & $\mathrm{X}$ & $X$ & $X$ \\
\hline
\end{tabular}


Tabela 1. Continuação...

$\begin{array}{cccc}\text { Alto } & \text { Ribeira } & \text { Paraíba } & \text { Bacia } \\ \text { Paraná } & \text { de Iguape } & \text { do Sul } & \text { Litorânea }\end{array}$

Lebiasinidae

Nannostomus beckfordi Günther, 1872

Pyrrhulina australis Eigenmann \& Kennedy, 1903

Parodontidae

Apareiodon affinis (Steindachner, 1879)

Apareiodon ibitiensis Campos, 1944

Apareiodon piracicabae (Eigenmann, 1907)

Parodon moreirai Ingenito \& Buckup, 2005

Parodon nasus Kner, 1859

Prochilodontidae

Prochilodus lineatus (Valenciennes, 1836)

Prochilodus vimboides Kner, 1859 *

Gymnotiformes

Apteronotidae

Apteronotus albifrons (Linnaeus, 1766)

Apteronotus brasiliensis (Reinhardt, 1852)

Apteronotus caudimaculosus Santana, 2003

Sternarchella curvioperculata Godoy, 1968 *

Sternarchorhynchus britskii Campos-da-Paz, 2000

Tembeassu marauna Triques, 1988 *

Gymnotidae

Gymnotus carapo Linnaeus, 1758

Gymnotus inaequilabiatus (Valenciennes, 1842)

Gymnotus pantanal Fernandes, Albert, Daniel-Silva, Lopes, Crampton \& AlmeidaToledo, 2005

Gymnotus pantherinus (Steindachner, 1908)

Gymnotus paraguensis Albert \& Crampton, 2003

Gymnotus sylvius Albert \& Fernandes-Matioli, 1999

$\begin{array}{llll}- & - & - & -\end{array}$

$\mathrm{X}$

$\mathrm{X}$

$\mathrm{X}$

$\mathrm{X}$

$\mathrm{X}$

$\mathrm{X}$

$\mathrm{X}$

$\mathrm{X}$

$\mathrm{X}$

$\mathrm{X}$

ypopomidae

Brachyhypopomus jureiae Triques \& Khamis, 2003 *

Brachyhypopomus pinnicaudatus (Hopkins, Comfort, Bastian \& Bass, 1990)

Rhamphichthyidae

Rhamphichthys hahni (Meinken, 1937)

Sternopygidae

Eigenmannia trilineata López \& Castello, 1966

Eigenmannia virescens (Valenciennes, 1847)

Sternopygus macrurus (Bloch \& Schneider, 1801)

$\mathrm{X}$

$\mathrm{X}$

$\mathrm{X}$

$\mathrm{X}$

$\mathrm{X}$

$\mathrm{X}$

$\mathrm{X}$

$\mathrm{X}$

$\mathrm{X}$

$\mathrm{X}$

$\mathrm{X}$

$\mathrm{X}$

X

$\mathrm{X}$

$\mathrm{X}$

$\mathrm{X}$

X

$\mathrm{X}$

$\mathrm{X}$

$\mathrm{X}$

$\mathrm{X}$

$\mathrm{X}$

Siluriformes

Aspredinidae

Bunocephalus larai Ihering, 1930 *

$\mathrm{X}$

Auchenipteridae

Ageneiosus militaris Valenciennes, 1836

Auchenipterus osteomystax (Miranda-Ribeiro, 1918)

Glanidium cesarpintoi Ihering, 1928

Glanidium melanopterum Miranda-Ribeiro, 1918

Tatia neivai (Ihering, 1930)

Trachelyopterus coriaceus Valenciennes, 1840

Trachelyopterus galeatus (Linnaeus, 1766)

Callichthyidae 
Tabela 1. Continuação...

\begin{tabular}{|c|c|c|c|c|}
\hline & $\begin{array}{c}\text { Alto } \\
\text { Paraná }\end{array}$ & $\begin{array}{c}\text { Ribeira } \\
\text { de Iguape }\end{array}$ & $\begin{array}{c}\text { Paraíba } \\
\text { do Sul }\end{array}$ & $\begin{array}{c}\text { Bacia } \\
\text { Litorânea }\end{array}$ \\
\hline Aspidoras fuscoguttatus Nijssen \& Isbrücker, 1976 & $\mathrm{X}$ & - & - & - \\
\hline Aspidoras lakoi Miranda-Ribeiro, 1949) & $\mathrm{X}$ & - & - & - \\
\hline Callichthys callichthys (Linnaeus, 1758) & $\mathrm{X}$ & $\mathrm{X}$ & $\mathrm{X}$ & - \\
\hline Corydoras aeneus (Gill, 1858) & $\mathrm{X}$ & - & - & - \\
\hline Corydoras difluviatilis Britto \& Castro, 2002 & $\mathrm{X}$ & - & - & - \\
\hline Corydoras ehrhardti Steindachner, 1910 & $\mathrm{X}$ & $\mathrm{X}$ & - & - \\
\hline Corydoras flaveolus Ihering, 1911 & $\mathrm{X}$ & - & - & - \\
\hline Corydoras nattereri Steindachner, 1877 & $\mathrm{X}$ & $\mathrm{X}$ & - & - \\
\hline Hoplosternum littorale (Hancock, 1828) & $\mathrm{X}$ & $\mathrm{X}$ & $\mathrm{X}$ & $\mathrm{X}$ \\
\hline Lepthoplosternum pectorale (Boulenger, 1895) & $\mathrm{X}$ & - & - & - \\
\hline Megalechis personata (Ranzani, 1841) & $\mathrm{X}$ & - & - & - \\
\hline Scleromystax barbatus (Quoy \& Gaimard, 1824) & - & $\mathrm{X}$ & $\mathrm{X}$ & $\mathrm{X}$ \\
\hline Scleromystax macropterus Regan, $1913 *$ & - & $X$ & - & $\mathrm{X}$ \\
\hline Scleromystax prionotos Nijssen \& Isbrücker, $1980 *$ & - & $X$ & - & $\mathrm{X}$ \\
\hline \multicolumn{5}{|l|}{ Cetopsidae } \\
\hline Cetopsis gobioides Kner, 1857 & $\mathrm{X}$ & $X$ & - & - \\
\hline \multicolumn{5}{|l|}{ Clariidae } \\
\hline Clarias gariepinus (Burchell, 1840) & $\mathrm{X}$ & $\mathrm{X}$ & - & - \\
\hline \multicolumn{5}{|l|}{ Doradidae } \\
\hline Platydoras armatulus(Valenciennes, 1840) & $X$ & - & - & - \\
\hline Pterodoras granulosus (Valenciennes, 1821) & $\mathrm{X}$ & - & - & - \\
\hline Oxydoras eigenmanni Boulenger, 1895 & $\mathrm{X}$ & - & - & - \\
\hline Rhinodoras dorbignyi (Kner, 1855) & $\mathrm{X}$ & - & - & - \\
\hline Trachydoras paraguayensis (Eigenmann \& Ward, 1907) & $\mathrm{X}$ & - & - & - \\
\hline \multicolumn{5}{|l|}{ Heptapteridae } \\
\hline Acentronichthys leptos Eigenmann \& Eigenmann, 1889 & - & $\mathrm{X}$ & - & $\mathrm{X}$ \\
\hline Cetopsorhamdia iheringi Schubart \& Gomes, 1959 & $\mathrm{X}$ & - & - & - \\
\hline Chasmocranus brachynema Gomes \& Schubart, 1958 * & $X$ & - & - & - \\
\hline Chasmocranus lopezi (Miranda Ribeiro, 1968) & - & $X$ & - & - \\
\hline Heptapterus multiradiatus Ihering, $1907 *$ & $\mathrm{X}$ & - & - & - \\
\hline Imparfinis borodini Mees \& Cala, 1989 & $X$ & - & - & - \\
\hline Imparfinis mirini Haseman, 1911 & $\mathrm{X}$ & - & - & - \\
\hline Imparfinis minutus (Lütken, 1874) & - & $X$ & $\mathrm{X}$ & - \\
\hline Imparfinis piperatus Eigenmann \& Norris, 1900 & $X$ & - & - & - \\
\hline Imparfinis schubarti (Gomes, 1956) & $\mathrm{X}$ & - & - & - \\
\hline Phenacorhamdia tenebrosa (Schubart, 1964) & $\mathrm{X}$ & - & - & - \\
\hline Pimelodella avanhandavae Eigenmann, 1917 & $\mathrm{X}$ & - & - & - \\
\hline Pimelodella boschmai Van der Stigchel, 1964 & $\mathrm{X}$ & - & - & - \\
\hline Pimelodella eigenmanni (Boulenger, 1891) & - & - & $\mathrm{X}$ & - \\
\hline Pimelodella gracilis (Valenciennes, 1835) & $\mathrm{X}$ & - & - & - \\
\hline Pimelodella kronei (Ribeiro, 1907)* & - & $\mathrm{X}$ & - & - \\
\hline Pimelodella lateristriga (Müller \& Troschel, 1849) & - & - & $\mathrm{X}$ & - \\
\hline Pimelodella meeki Eigenmann, 1910 & $\mathrm{X}$ & - & - & - \\
\hline \multicolumn{5}{|l|}{ Pimelodella taenioptera Miranda-Ribeiro, 1918} \\
\hline Pimelodella rudolphi Miranda-Ribeiro, 1918 & $\mathrm{X}$ & - & - & - \\
\hline Pimelodella transitoria (Ribeiro, 1907) & - & $\mathrm{X}$ & - & - \\
\hline Rhamdella longipinnis Borodin, 1927 & $X$ & - & - & - \\
\hline Rhamdia quelen (Quoy \& Gaimard, 1824) & $X$ & $X$ & $X$ & $X$ \\
\hline Rhamdioglanis frenatus Ihering, 1907 & - & - & - & $\mathrm{X}$ \\
\hline
\end{tabular}


Tabela 1. Continuação...

Rhamdiopsis microcephala (Lütken, 1874)

Taunaya bifasciata (Eigenmann \& Norris, 1900) *

Loricariidae

Ancistrus cirrhosus (Valenciennes, 1836)

Ancistrus multispinis (Regan, 1912)

Corumbataia cuestae Britski, 1997 *

Farlowella hahni Meinke, 1937

Farlowella oxyrhyncha (Kner, 1853)

Harttia carvalhoi Miranda-Ribeiro, 1939

Harttia gracilis Oyakawa, 1993 *

Harttia kronei Miranda-Ribeiro, 1908

Harttia loricariformis Steindachner, 1876 *

Hemipsilichthys gobio (Lütken, 1874) *

Hisonotus depressicauda (Miranda-Ribeiro, 1918)

Hisonotus depressinotus (Miranda-Ribeiro, 1918)

Hisonotus francirochai (Ihering, 1928)

Hisonotus leucofrenatus (Miranda-Ribeiro, 1908)

Hisonotus insperatus Britski \& Garavello, 2003

Hisonotus notatus Eigenmann \& Eigenmann, 1889

Hisonotus paulinus (Regan, 1908)

Hypostomus affinis (Steindachner, 1877)

Hypostomus agna (Miranda-Ribeiro, 1907)

Hypostomus albopunctatus (Regan, 1908)

Hypostomus ancistroides (Ihering, 1911)

Hypostomus brevis (Nichols, 1919)

Hypostomus fluviatilis (Schubart, 1964)

Hypostomus hermanni (Ihering, 1905)

Hypostomus iheringi (Regan, 1908)

Hypostomus interruptus (Miranda-Ribeiro, 1918)

Hypostomus lexi (Ihering, 1911)

Hypostomus luetkeni (Steindachner, 1876)

Hypostomus margaritifer (Regan, 1908)

Hypostomus meleagris (Marini; Nichols \& La Monte, 1933)

Hypostomus microstomus Weber, 1987

Hypostomus nigromaculatus (Schubart, 1967)

Hypostomus paulinus (Ihering, 1905)

Hypostomus regani (Ihering, 1905)

Hypostomus scaphyceps (Nichols, 1919)

Hypostomus strigaticeps (Regan, 1908)

Hypostomus tapijara Oyakawa, Akama \& Zanata, 2005

Hypostomus ternetzi (Boulenger, 1895)

Hypostomus tietensis (Ihering, 1905)

Hypostomus topavae (Godoy, 1969)

Hypostomus variipictus (Ihering, 1911)

Isbrueckerichthys alipionis (Gosline, 1947)

Isbrueckerichthys duseni (Miranda-Ribeiro, 1907) *

Isbrueckerichthys epakmos Pereira \& Oyakawa, 2003 *

Kronichthys heylandi (Boulenger, 1900)

Kronichthys lacerta (Nichols, 1919)

Kronichthys subteres Miranda-Ribeiro, 1908

\begin{tabular}{cccc} 
Alto & Ribeira & Paraíba & Bacia \\
Paraná & de Iguape & do Sul & Litorânea \\
\hline
\end{tabular}

$\begin{array}{cccc}- & X & - & - \\ X & - & - & - \\ X & - & X & X\end{array}$

X

$\mathrm{X}$

$\mathrm{X}$

$\mathrm{X}$

$-$ 
Tabela 1. Continuação...

\begin{tabular}{|c|c|c|c|c|}
\hline & $\begin{array}{c}\text { Alto } \\
\text { Paraná }\end{array}$ & $\begin{array}{c}\text { Ribeira } \\
\text { de Iguape }\end{array}$ & $\begin{array}{c}\text { Paraíba } \\
\text { do Sul }\end{array}$ & $\begin{array}{c}\text { Bacia } \\
\text { Litorânea }\end{array}$ \\
\hline Lampiella gibbosa (Miranda-Ribeiro, 1908) & - & $\mathrm{X}$ & - & - \\
\hline Loricaria piracicabae Ihering, 1907 & $\mathrm{X}$ & - & - & - \\
\hline Loricaria simillima Regan, 1904 & $\mathrm{X}$ & - & - & - \\
\hline Loricariichthys castaneus (Casteulnau, 1855) & - & $\mathrm{X}$ & $\mathrm{X}$ & - \\
\hline Loricariichthys platymetopon Isbrücker \& Nijssen, 1979 & $\mathrm{X}$ & - & - & - \\
\hline Loricariichthys rostratus Reis \& Pereira, 2000 & $\mathrm{X}$ & - & - & - \\
\hline Megalancistrus parananus (Peters, 1881) & $\mathrm{X}$ & - & - & - \\
\hline Neoplecostomus paranensis Langeani, $1990 *$ & $\mathrm{X}$ & - & - & - \\
\hline Neoplecostomus microps (Steindachner, 1876) & - & - & $\mathrm{X}$ & - \\
\hline Neoplecostomus ribeirensis Langeani, 1990 & - & $\mathrm{X}$ & - & - \\
\hline Neoplecostomus seleneae Zawadzki, Pavanelli \& Langeani, 2008 * & $\mathrm{X}$ & - & - & - \\
\hline Otocinclus affinis Steindachner, 1877 & - & $\mathrm{X}$ & - & - \\
\hline Otothyris juquiae Garavello, Britski \& Schaefer, 1998 * & - & $\mathrm{X}$ & - & - \\
\hline Otothyropsis marapoama Ribeiro; Carvalho \& Melo, 2005 & $\mathrm{X}$ & - & - & - \\
\hline Pareiorhina brachyrhyncha Chamon, Aranda \& Buckup, 2005 * & - & - & $\mathrm{X}$ & - \\
\hline \multicolumn{5}{|l|}{ Pareiorhina carrancas Bockmann \& Ribeiro, 2003} \\
\hline Pareiorhina rudolphi (Miranda-Ribeiro, 1911) * & - & - & $\mathrm{X}$ & - \\
\hline Parotocinclus maculicauda (Steindachner, 1877) & - & $\mathrm{X}$ & - & $\mathrm{X}$ \\
\hline Pogonopoma parahybae (Steindachner, 1877) & - & - & $\mathrm{X}$ & - \\
\hline Proloricaria lentiginosa (Isbrücker, 1979) & $X$ & - & - & - \\
\hline Proloricaria prolixa (Isbrücker \& Nijssen, 1978) & $\mathrm{X}$ & - & - & - \\
\hline Pseudotocinclus juquiae Takako, Oliveira \& Oyakawa, 2005 * & - & $\mathrm{X}$ & - & - \\
\hline Pseudotocinclus parahybae Takako, Oliveira \& Oyakawa, $2005 *$ & - & - & $\mathrm{X}$ & - \\
\hline Pseudotocinclus tietensis (Ihering, 1907) * & $\mathrm{X}$ & - & - & - \\
\hline Pseudotothyris obtusa (Ribeiro, 1911) & - & $\mathrm{X}$ & - & $\mathrm{X}$ \\
\hline Pterygoplichthys anisitsi Eigenmann \& Kennedy, 1903 & $\mathrm{X}$ & - & - & - \\
\hline Rhinelepis aspera Spix \& Agassiz, 1829 & $\mathrm{X}$ & - & - & - \\
\hline Rineloricaria kronei (Miranda-Ribeiro, 1911) & - & $\mathrm{X}$ & - & - \\
\hline Rineloricaria latirostris (Boulenger, 1900) & $\mathrm{X}$ & - & - & - \\
\hline Rineloricaria lima (Kner, 1853) & - & $\mathrm{X}$ & $\mathrm{X}$ & - \\
\hline Rineloricaria nigricauda (Regan, 1904) & - & - & $\mathrm{X}$ & - \\
\hline Rineloricaria pentamaculata Langeani \& Araújo, 1994 & $X$ & - & - & - \\
\hline Rineloricaria steindachneri (Regan, 1904) & - & - & $\mathrm{X}$ & - \\
\hline Schizolecis guntheri (Ribeiro, 1918) & - & $X$ & - & $X$ \\
\hline \multicolumn{5}{|l|}{ Pimelodidae } \\
\hline Hemisorubim platyrhynchos (Valenciennes, 1840) * & $\mathrm{X}$ & - & - & - \\
\hline Hypophthalmus edentatus Spix \& Agassiz, 1829 & $\mathrm{X}$ & - & - & - \\
\hline Iheringichthys labrosus (Lütken, 1874) & $\mathrm{X}$ & - & - & - \\
\hline Pimelodus fur (Lütken, 1874) & $X$ & - & - & - \\
\hline Pimelodus maculatus La Cepède, 1803 & $\mathrm{X}$ & $X$ & $X$ & $X$ \\
\hline Pimelodus microstoma Steindachner, 1877 & $\mathrm{X}$ & - & - & - \\
\hline Pimelodus paranaensis Britski \& Langeani, $1988 *$ & $\mathrm{X}$ & - & - & - \\
\hline Pimelodus platicirris Borodin, 1927 & $\mathrm{X}$ & - & - & - \\
\hline Pinirampus pirinampu (Spix \& Agassiz, 1829) & $\mathrm{X}$ & - & - & - \\
\hline Pseudoplatystoma corruscans (Spix \& Agassiz, 1829) * & $\mathrm{X}$ & - & - & - \\
\hline Pseudoplatystoma fasciatum (Linnaeus, 1766) & $\mathrm{X}$ & - & - & - \\
\hline Sorubim lima (Bloch \& Schneider, 1801) & $\mathrm{X}$ & - & - & - \\
\hline Steindachneridion parahybae Eigenmann \& Eigenmann, $1888 *$ & - & - & $\mathrm{X}$ & - \\
\hline Steindachneridion punctatum (Miranda-Ribeiro, 1918) * & $X$ & - & - & - \\
\hline
\end{tabular}


Tabela 1. Continuação...

\begin{tabular}{|c|c|c|c|c|}
\hline & $\begin{array}{c}\text { Alto } \\
\text { Paraná }\end{array}$ & $\begin{array}{c}\text { Ribeira } \\
\text { de Iguape }\end{array}$ & $\begin{array}{c}\text { Paraíba } \\
\text { do Sul }\end{array}$ & $\begin{array}{c}\text { Bacia } \\
\text { Litorânea }\end{array}$ \\
\hline Steindachneridion scriptum (Miranda-Ribeiro, 1918) * & $\mathrm{X}$ & - & - & - \\
\hline Zungaro jahu (Ihering, 1898)* & $\mathrm{X}$ & - & - & - \\
\hline \multicolumn{5}{|l|}{ Pseudopimelodidae } \\
\hline Microglanis cottoides (Boulenger, 1891) & - & $\mathrm{X}$ & - & - \\
\hline Microglanis garavelloi Shibatta \& Benine, 2005 & $\mathrm{X}$ & - & - & - \\
\hline Microglanis parahybae (Steindachner, 1880) & - & - & $\mathrm{X}$ & - \\
\hline Pseudopimelodus mangurus (Valenciennes, 1835) * & $\mathrm{X}$ & - & - & - \\
\hline Pseudopimelodus aff. pulcher (Boulenger, 1887) & $\mathrm{X}$ & - & - & - \\
\hline \multicolumn{5}{|l|}{ Trichomycteridae } \\
\hline Homodiaetus graciosa Koch, $2002 *$ & - & $\mathrm{X}$ & - & - \\
\hline Ituglanis proops (Miranda-Ribeiro, 1908) & - & $X$ & - & - \\
\hline Ituglanis parahybae (Eigenmann, 1918) & - & $\mathrm{X}$ & $X$ & - \\
\hline Ituglanis sp. & $X$ & - & - & - \\
\hline Listrura camposi (Miranda-Ribeiro, 1957) * & - & $\mathrm{X}$ & - & - \\
\hline Listrura nematoperyx de Pinna, 1988 & - & - & - & $\mathrm{X}$ \\
\hline Listrura picinguabae Villa-Verde \& Costa, $2006 *$ & - & - & - & $\mathrm{X}$ \\
\hline Microcambeva ribeirae Costa, Lima \& Bizerril, 2004 & - & $\mathrm{X}$ & - & - \\
\hline Paravandellia oxyptera Miranda Ribeiro, 1912 & $\mathrm{X}$ & - & - & - \\
\hline Parastegophilus paulensis (Miranda Ribeiro, 1918) & $\mathrm{X}$ & - & - & - \\
\hline Trichogenes longipinnis Britski \& Ortega, $1986 *$ & - & - & - & $\mathrm{X}$ \\
\hline Trichomycterus alternatus (Eigenmann, 1917) & - & - & $\mathrm{X}$ & - \\
\hline Trichomycterus brasiliensis Reinhardt, 1873 & $\mathrm{X}$ & - & - & - \\
\hline Trichomycterus davisi (Haseman, 1911) & - & $\mathrm{X}$ & - & $\mathrm{X}$ \\
\hline Trichomycterus diabolus Bockmann; Casatti \& de Pinna, 2004 & $\mathrm{X}$ & - & - & - \\
\hline Trichomycterus iheringi (Eigenmann, 1917) & $\mathrm{X}$ & $\mathrm{X}$ & $\mathrm{X}$ & $\mathrm{X}$ \\
\hline Trichomycterus immaculatus (Eigenmann \& Eigenmann, 1889) & - & - & $\mathrm{X}$ & - \\
\hline Trichomycterus itatiayae (Miranda-Ribeiro, 1906) & - & - & $X$ & - \\
\hline Trichomycterus jacupiranga Wosiacki \& Oyakawa, 2005 & - & $X$ & - & - \\
\hline Trichomycterus maracaya Bockmann \& Sazima, 2004 & $\mathrm{X}$ & - & - & - \\
\hline Trichomycterus mimonha Costa, 1992 & - & - & $X$ & - \\
\hline Trichomycterus paolence (Eigenmann, 1917) * & $X$ & - & - & $\mathrm{X}$ \\
\hline \multicolumn{5}{|l|}{ Trichomycterus pauciradiatus Alencar \& Costa, 2006} \\
\hline Trichomycterus tupinamba Wosiacki \& Oyakawa, 2005 & - & $X$ & - & - \\
\hline Trichomycterus triguttatus (Eigenmann, 1918) & - & - & $X$ & - \\
\hline Trichomycterus zonatus (Eigenmann, 1918) & - & $\mathrm{X}$ & - & $\mathrm{X}$ \\
\hline \multicolumn{5}{|l|}{ Cyprinodontiformes } \\
\hline \multicolumn{5}{|l|}{ Poeciliidae } \\
\hline Cnesterodon iguape Lucinda, $2005 *$ & - & $\mathrm{X}$ & - & - \\
\hline Phalloceros harpagos Lucinda, 2008 & $\mathrm{X}$ & $\mathrm{X}$ & $\mathrm{X}$ & $\mathrm{X}$ \\
\hline Phalloceros lucenorum Lucinda, 2008 & - & $\mathrm{X}$ & - & - \\
\hline Phalloceros reisi Lucinda, 2008 & $\mathrm{X}$ & $\mathrm{X}$ & - & $\mathrm{X}$ \\
\hline Phalloceros tupinamba Lucinda, 2008 & - & - & - & $\mathrm{X}$ \\
\hline Phalloptychus januarius (Hensel, 1868) & - & - & - & $\mathrm{X}$ \\
\hline Phallotorynus fasciolatus Henn, 1916 * & $X$ & - & $X$ & - \\
\hline Phallotorynus jucundus Ihering, $1931 *$ & $X$ & - & - & - \\
\hline \multicolumn{5}{|l|}{ Poecilia reticulata Peters, 1859} \\
\hline Poecilia vivipara Bloch \& Schneider, 1801 & $\mathrm{X}$ & $\mathrm{X}$ & $\mathrm{X}$ & - \\
\hline Hiphophorus helleri Heckel, 1848 & $X$ & - & - & - \\
\hline Xiphophorus maculatus (Günther, 1866) & $\mathrm{X}$ & - & - & - \\
\hline
\end{tabular}


Tabela 1. Continuação...

\begin{tabular}{|c|c|c|c|c|}
\hline & $\begin{array}{c}\text { Alto } \\
\text { Paraná }\end{array}$ & $\begin{array}{c}\text { Ribeira } \\
\text { de Iguape }\end{array}$ & $\begin{array}{c}\text { Paraíba } \\
\text { do Sul }\end{array}$ & $\begin{array}{c}\text { Bacia } \\
\text { Litorânea }\end{array}$ \\
\hline \multicolumn{5}{|l|}{ Rivulidae } \\
\hline Campellolebias dorsimaculatus Costa, Lacerda \& Brasil, $1989 *$ & - & $\mathrm{X}$ & - & - \\
\hline Campellolebias intermedius Costa \& De Luca, 2006 * & - & $\mathrm{X}$ & - & - \\
\hline Kryptolebias caudomarginatus (Seegers, 1984) & - & - & - & $\mathrm{X}$ \\
\hline Kryptolebias ocellatus (Hensel, 1868) & - & - & - & $\mathrm{X}$ \\
\hline Leptolebias aureoguttatus (Cruz, 1974) * & - & $\mathrm{X}$ & - & $\mathrm{X}$ \\
\hline Leptolebias itanhaensis Costa, $2008 *$ & - & - & - & $\mathrm{X}$ \\
\hline Rivulus apiamici Costa, 1989 & $\mathrm{X}$ & - & - & - \\
\hline Rivulus santensis Köhler, 1906 & $\mathrm{X}$ & $\mathrm{X}$ & - & $\mathrm{X}$ \\
\hline \multicolumn{5}{|l|}{ Synbranchiformes } \\
\hline \multicolumn{5}{|l|}{ Synbranchidae } \\
\hline Synbranchus marmoratus Bloch, 1795 & $\mathrm{X}$ & $\mathrm{X}$ & $\mathrm{X}$ & - \\
\hline \multicolumn{5}{|l|}{ Perciformes } \\
\hline \multicolumn{5}{|l|}{ Blenniidae } \\
\hline Lupinoblennius paivai (Pinto, 1958) & - & - & - & $X$ \\
\hline \multicolumn{5}{|l|}{ Centrarchidae } \\
\hline Micropterus salmoides (La Cepède, 1802) & $\mathrm{X}$ & - & - & - \\
\hline \multicolumn{5}{|l|}{ Cichlidae } \\
\hline Astronotus crassipinnis Heckel, 1840 & $\mathrm{X}$ & - & - & - \\
\hline Australoheros facetus (Jenyns, 1842) & - & - & $\mathrm{X}$ & - \\
\hline Australoheros ribeirae Ottoni, Oyakawa \& Costa, 2008 & - & $\mathrm{X}$ & - & - \\
\hline Cichla kelberi Kullander \& Ferreira, 2006 & $\mathrm{X}$ & - & - & - \\
\hline Cichla piquiti Kullander \& Ferreira, 2006 & $\mathrm{X}$ & - & - & - \\
\hline Australoheros facetus (Jenyns, 1842) & $\mathrm{X}$ & - & - & - \\
\hline Cichlasoma paranaense Kullander, 1983 & $\mathrm{X}$ & - & - & - \\
\hline Crenicichla britskii Kullander, 1982 & $\mathrm{X}$ & - & - & - \\
\hline Crenicichla haroldoi Luengo \& Britski, 1974 & $\mathrm{X}$ & - & - & - \\
\hline Crenicichla iguapina Kullander \& Lucena, 2006 & - & $\mathrm{X}$ & - & - \\
\hline Crenicichla jaguarensis Haseman, 1911 & $\mathrm{X}$ & - & - & - \\
\hline Crenicichla jupiaiensis Britski \& Luengo, 1968 * & $\mathrm{X}$ & - & - & - \\
\hline Crenicichla lacustris (Castelnau, 1855) & - & - & $\mathrm{X}$ & - \\
\hline Geophagus brasiliensis (Quoy \& Gaimard, 1824) & $\mathrm{X}$ & - & $X$ & $\mathrm{X}$ \\
\hline Geophagus iporanguensis Haseman, 1911 & - & $X$ & - & - \\
\hline Geophagus proximus (Castelnau, 1855) & $X$ & - & - & - \\
\hline Oreochromis niloticus (Linnaeus, 1758) & $\mathrm{X}$ & $\mathrm{X}$ & - & - \\
\hline Satanoperca pappaterra (Heckel, 1840) & $\mathrm{X}$ & - & - & - \\
\hline Tilapia rendalli (Boulenger, 1897) & $\mathrm{X}$ & $\mathrm{X}$ & - & - \\
\hline \multicolumn{5}{|l|}{ Eleotridae } \\
\hline Dormitator maculatus (Bloch, 1792) & - & - & - & $\mathrm{X}$ \\
\hline Eleotris pisonis (Gmelin, 1789) & - & - & - & $\mathrm{X}$ \\
\hline Guavina guavina (Valenciennes, 1837) & - & - & - & $\mathrm{X}$ \\
\hline \multicolumn{5}{|l|}{ Gobiidae } \\
\hline Awaous tajasica (Lichtenstein, 1822) & - & - & - & $\mathrm{X}$ \\
\hline Ctenogobius shufeldti (Jordan \& Eigenmann, 1887) & - & - & - & $\mathrm{X}$ \\
\hline \multicolumn{5}{|l|}{ Sciaenidae } \\
\hline Plagioscion squamosissimus (Heckel, 1840) & $\mathrm{X}$ & - & - & - \\
\hline \multicolumn{5}{|l|}{ Pleuronectiformes } \\
\hline \multicolumn{5}{|l|}{ Achiridae } \\
\hline Catathyridium jenynsii (Günther, 1862) & $\mathrm{X}$ & - & - & - \\
\hline
\end{tabular}


Observando-se novamente a Tabela 1, alguns aspectos merecem ser ressaltados. Das quatro espécies que compõem o gênero Spintherobolus, três ocorrem em São Paulo e as três estão ameaçadas. Todas as três espécies do gênero Pseudotocinclus, cada uma delas restrita à uma bacia hidrográficas do estado, estão sob diferentes graus de ameaça. Steindachneridion, gênero composto por seis espécies, possui três representantes no estado; duas dessas - S. punctatum (Miranda-Ribeiro, 1918) e S. scriptum (Miranda-Ribeiro, 1918) do Alto Paraná estão ameaçadas e S. parahybae (Steindachner, 1877), da bacia do Paraíba do Sul, foi considerada como regionalmente extinta no Estado de São Paulo. Populações naturais de Brycon insignis Steindachner, 1877, que na década de 1950 foi um importante recurso pesqueiro na porção paulista da bacia do Rio Paraíba do Sul, e B. nattereri Günther, 1864 do Alto Paraná, estão provavelmente extintas no estado (Oyakawa et al. 2009).

Mesmo sendo a mais extensa bacia hidrográfica do Estado de São Paulo, o Alto Paraná como um todo, possui uma das ictiofaunas da América do Sul melhor conhecida e estudadas; apesar deste fato, o número de espécies ainda está longe de representar a realidade, uma vez que a curva de acúmulo de espécie não mostra nenhuma tendência de estabilização, e diversas descobertas futuras de novos táxons são esperadas na bacia (Langeani et al. 2007). A segunda bacia melhor estudada é a do Ribeira de Iguape, sendo o número estimado de espécies, cerca de 100, bastante fidedigno. Entretanto, aqui também existem algumas espécies novas e também um gênero novo da família Trichomycteridae já descobertas, mas que ainda não foram descritas formalmente. A bacia do Paraíba do Sul e a bacia litorânea ainda são praticamente terra incógnita na sua porção paulista, uma vez que ainda não foram objetos de inventariação sistemáticos como as duas outras bacias.

Pelo exposto acima fica claro que muito trabalho de coleta e de descrição das novas espécies ainda necessitam ser realizadas. No que tange às coletas, no Alto Paraná ambientes profundos de calha nunca foram amostradas, bem como ambientes como áreas marginais e de alagadiços de rios e represas (Langeani et al. 2007). Duas espécies de Gymnotiformes, ameaçadas de extinção, Sternarchorhynchus britskii Campos da Paz, 2000 e Tembeassu marauna são conhecidas de coletas feitas em ensecadeiras, no leito do Rio Paraná em locais de rochas e corredeiras, durante a construção da usina hidrelétrica de Ilha Solteira, nos anos de 1965 e 1972. Atualmente existem outros exemplares da primeira espécie coletadas em outras localidades como o Rio Grande na divisa entre São Paulo e Minas Gerais (Campos-da-Paz 2005). Tembeassu marauna Triques, 1998, por outro lado, até o momento só é conhecida dos três exemplares que serviram de base para a sua descrição em 1998.

Na bacia do Alto Tietê, em áreas intensamente urbanizadas dos municípios de Embu das Artes, Itapecerica da Serra e São Paulo, coletas recentes revelaram a presença de pequenas populações de espécies raras, espécies ameaçadas de extinção e também de espécies que nunca antes haviam sido registradas no Alto Tietê. Alguns exemplos são: Hyphessobrycon flammeus Myers, 1924, espécie ameaçada de extinção e conhecida apenas da baixada fluminense; Rivulus santensis Köhler, 1906, conhecida apenas da bacia litorânea; Trichomycterus paolence (Eigenmann, 1917), também ameaçada de extinção, conhecida anteriormente somente da região de Paranapiacaba no município de Santo André; e Ituglanis sp. gênero nunca antes referido para o Alto Tietê. Outro exemplo bastante ilustrativo sobre a necessidade de coletas em diferentes ambientes é dado pelo trabalho de Serra et al. (2007) no Rio Itatinga, afluente do Rio Itapanhaú que pertence à bacia litorânea. O Rio Itatinga, apesar de ser um afluente do Itapanhaú, que pertence à bacia litorânea, tem suas nascentes no topo da serra da região do planalto atlântico paulista. Neste rio os autores identificaram uma grande população de
Coptobrycon bilineatus (Ellis, 1911), espécie ameaçada de extinção, da qual os únicos registros anteriores datavam do longínquo ano de 1908 na região de Paranapiacaba. Além desta espécie foram registradas também Pseudotocinclus tietensis (Ihering, 1907), Glandulocauda melanopleura (Ellis, 1911) e Taunayia bifasciata (Eigenmann \& Norris, 1900), todas também ameaçadas de extinção, além de Trichomycterus iheringi (Eigenmann, 1917) identificado pelos autores como Trichomycterus sp., todos típicos representantes da ictiofauna do Alto Tietê. O trabalho de Ribeiro et al. (2006) também é bastante ilustrativo ao registrar na parte superior do Rio Guaratuba, outro rio litorâneo cujas cabeceiras também estão localizadas no planalto atlântico, espécies como Astyanax paranae Eigenmann, 1914, Glandulocauda melanopleura (Ellis, 1911), Characidium oiticicai Travasso, 1967e Trichomycterus paolence (Eigenmann, 1917), também anteriormente conhecidos por só ocorrerem no Alto Tietê. Trabalhos de inventariação ainda não finalizados parecem indicar o mesmo padrão na bacia do Rio Capivari-Monos, localizado na APA Capivari-Monos, na zona sul do município de São Paulo.

Durante as reuniões para a elaboração do Livro Vermelho da Fauna Ameaçada de Extinção do Estado de São Paulo", um dos integrantes do grupo de trabalho relatou a ocorrência de Rachoviscus crassiceps Myers, 1926, em riachos de águas pretas do município de Itanhaém. Anteriormente esta espécie só era conhecida oficialmente dos riachos litorâneos do município de Guaratuba no estado do Paraná. Posteriormente uma equipe do Museu de Zoologia da Universidade de São Paulo logrou coletar alguns exemplares da espécie que estão depositadas na coleção de peixes deste museu.

Na bacia do Rio Paraíba do Sul há outro exemplo do quanto ainda é falha a inventariação da ictiofauna do estado, pois recentemente foi registrada a ocorrência, em um dos braços a represa de Paraibuna, de Pogonopoma parahybae (Steindachner, 1877), espécie ameaçada de extinção, conhecida anteriormente só das porções carioca e mineira do Paraíba do Sul.

Entre os vários trabalhos de descrição de espécies novas do estado, três merecem ser destacados. Garutti \& Britski (2000) descrevem Astyanax altiparanae e fazem uma síntese a respeito das sete espécies do gênero que ocorrem no Alto Paraná. Astyanax altiparanae é espécie amplamente distribuída em todo o Alto Paraná mas era identificada, anteriormente à este trabalho, como Astyanax bimaculatus (Linnaeus, 1758), cuja localidade-tipo seria provavelmente no Suriname. Segundo os autores, análises preliminares indicariam que formas de Astyanax do grupo bimaculatus, portadoras de determinadas características como nesta espécie, ocorreriam somente na bacia Amazônica e ao norte desta; não ocorrendo, portanto em nenhuma bacia hidrográfica de São Paulo. Ainda segundo esses mesmos autores, Astyanax eigenmanniorum (Cope, 1894), descrita originalmente do Rio Grande do Sul e posteriormente assinalada em outras bacias, inclusive em São Paulo, seria também constituída de um complexo de espécies, que à semelhança de "A. bimaculatus", deverão ser desmembradas em várias espécies quando se realizarem análises mais criteriosas das mesmas. Confirmando esta afirmação, Vari \& Castro (2007) descrevem Astyanax bockmanni com base no exame de grande número de exemplares coletados no Alto Paraná, inclusive em São Paulo, todos identificados anteriormente como Astyanax eigenmanniorum (Cope, 1894).

Lucinda (2008) descreve 21 espécies novas de Phalloceros, até então tido como um gênero monotípico e com ampla distribuição nos rios do Sul e Sudeste do Brasil. O gênero, apesar de bem conhecido sob vários aspectos como ecologia, anatomia e embriologia, era muito pouco estudado quanto à sua sistemática e taxonomia. Em São Paulo, o autor reconhece quatro espécies novas, sendo uma no Paraíba do Sul, duas no Alto Paraná e três no Ribeira de Iguape e na Bacia Litorânea. 
Os principais grupos ativos de pesquisa sobre peixes de água doce no estado de São Paulo encontram-se no Museu de Zoologia da Universidade de São Paulo (MZUSP), Laboratório de Ictiologia de Ribeirão Preto, Departamento de Biologia, Universidade de São Paulo, Ribeirão Preto (LIRP), Laboratório de Ictiologia, Departamento de Zoologia e Botânica, Universidade Estadual Paulista - campus de São José do Rio Preto (DZSJRP) e Coleção do Laboratório de Biologia e Genética de Peixes da Universidade Estadual Paulista - campus de Botucatu (LBP).

\section{Referências Bibliográficas}

BUCKUP, P.A., MENEZES, N.A. \& GHAZZI, M.S. (eds.). Catálogo das espécies de peixes de água doce do Brasil. Museu Nacional, Rio de Janeiro, 2007, 195p.

CAMPOS-DA-PAZ, R. 2005. Previously undescribed dental arrangement among eletric knifefishes, with comments on the taxonomic and conservation status of Tembeassu marauna Triques (Otophysi: Gymnotiformes: Apteronotidae). Neotrop. Ichthyol. 3(3):395-400.

CASTRO, R.M. C. \& MENEZES, N.A. 1998. Estudo diagnóstico da diversidade de peixes do estado de São Paulo. In Biodiversidade do estado de São Paulo, Brasil: síntese do conhecimento ao final do século XX. Vertebrados (C.A. Joly \& C.E.M. Bicudo, orgs.). Programa BIOTA/ FAPESP, São Paulo, v.6, p. 3-13.

ESCHMEYER, W.N. (ed.) Catalog of fishes electronic version (26 August 2010). http://research.calacademy.org/ichthyology/catalog/fishcatmain. asp

GARUTTI, V. \& BRITSKI, H.A. 2000. Descrição de uma espécie nova de Astyanax (Teleostei: Characidae) da bacia do Alto rio Paraná e considerações sobre as demais espécies do gênero na Bacia. Comunicações do Museu de Ciências e Tecnologia da PUCRS, Série Zoologia, Porto Alegre, 13:65-88.
INTERNACIONAL UNION FOR CONSERVATION OF NATURE AND NATURAL RESOURCES -IUCN. 2001. IUCN red list categories and criteria: version 3.1. IUCN Species Survival Comission. IUCN, Gland, Switzerland and Cambridge, UK, 30p.

LANGEANI, F., CASTRO, R.M.C., OYAKAWA, O.T., SHIBATTA, O.A., PAVANELLI, C.S. \& CASATTI, L. 2007. Diversidade da ictiofauna do Alto Rio Paraná: composição atual e perspectivas futuras. Biota Neotrop. 7(3):181-197.

LUCINDA, P. H. F. 2008. Systematics and biogeography of the genus Phalloceros Eigenmann, 1907 (Cyprinodontiformes: Poeciliidae: Poeciliinae), with description of twenty-one new species. Neotrop. Ichthyol. 6(2):113-158.

NELSON, J.S. 2006. Fishes of the World. 4th ed. John Wiley \& Sons, 601p.

OYAKAWA, T.O., MENEZES, N.A., SHIBATTA, O.A., LIMA, F.C.T., LANGEANI, F., PAVANELLI, C.S., NIELSEN, D.T.B., HILSDORF, A.W.S. 2009. Peixes de água doce. In Fauna ameaçada de extinção no Estado de São Paulo: vertebrados (P.M. Bressan, M.C.M. Kierulff \& A.M. Sugieda, eds.). Fundação Parque Zoológico de São Paulo, Secretaria do Meio Ambiente, São Paulo.

REIS, R.E., KULLANDER, S.O. \& FERRARIS. C.J. (eds.). 2003. Check list of the freshwater fishes of South and Central America. EDIPUCRS, Porto Alegre, 729p.

RIBEIRO, A.C., LIMA, F.C.T., RICCOMINI, C. \& MENEZES, N.A. 2006. Fishes of the Atlantic Rainforest of Boracéia: testimonies of the Quaternary fault reactivation within a Neoproterozoic tectonic province in Southeastern Brazil. Ichthyol. Explor. Freshw. 17(2):157-164.

SERRA, J.P., CARVALHO, F.R. \& LANGEANI, F. 2007. Ichthyofauna of the rio Itatinga in the Parque das Neblinas, Bertioga, São Paulo: composition and biogeography. Biota Neotrop. 7(1): http://www.biotaneotropica.org. $\mathrm{br} / \mathrm{v} 7 \mathrm{n} 1 / \mathrm{pt} / \mathrm{abstract}$ ?article+BN01707012007

VARI, R.P., CASTRO, R.M. C. 2007. New species of Astyanax (Ostariophysi: Characiformes: Characidae) from the Upper Rio Paraná System, Brazil. Copeia 1:150-162. 